\title{
Design of Bluetooth Automatic Check in System Without Operation Based on Cross Mobile Platform
}

\author{
Yiqin $\mathrm{BAO}^{\mathrm{a} 1}$ and Zhengtang $\mathrm{SUN}^{\mathrm{b}}$ \\ ${ }^{a}$ College of Information Engineering, Nanjing XiaoZhuang University, China \\ ${ }^{b}$ School of Computer Science and Technology, Heilongjiang University, China
}

\begin{abstract}
Because the traditional way of sign in is not to call the roll, or sign in on the paper list, or sign in through fingerprint recognition and face recognition, on the one hand, it is cumbersome and time-consuming, on the other hand, it is non-human. The update of roll call sign in is a hot topic at present, and many new schemes rush out. This paper introduces the automatic sign in system based on Bluetooth technology, which does not rely on the mobile phone operating system, does not need the mobile phone installation software, as long as you bring a mobile phone, you can realize automatic sign in. This paper designs and implements the automatic check-in system, and compares it with other ways, proving that it is an innovative check-in method.
\end{abstract}

Keywords. Automatic registration, bluetooth technology, JSON, SQLite

\section{Introduction}

At present, there are a total of more than 2000 colleges and universities in China. Many of them need to analyse and manage the data of the students who are always absent, so they need to count. Therefore, the attendance in a college is very important for the quality management. Now most of the colleges and universities have no difference with the past in the way of signing in, which is still a name called and the hands up, very troublesome and time-consuming. Sometimes teachers can according to their own experience, no teacher can do it in every class in usual, which leads a lot of students to produce fluky psychology. This will undoubtedly have a certain impact on the quality of teaching. To solve this problem, it is high time to change the traditional tedious way. There are a lot of new ways such as using a credit card, face recognition, fingerprint recognition, but either inconvenient, or high cost, most importantly is not humanized, so I need a new humanized way.

In recent years, many related technologies have been applied to check-in and roll call. Zhang et al. have studied the experimental check-in system based on RFID [1], Wang et al. have studied the mobile phone check-in system based on wireless router [2], and the roll call system based on face recognition [3-5]. However, due to these methods, on the one hand, the operation is cumbersome and time-consuming, on the other hand, it is not humanized and other weaknesses, so we hope to have a better way of roll call. Due to the popularity of mobile phones and the innovation of Bluetooth technology, it is feasible to use mobile phones to realize roll call through Bluetooth.

1 Corresponding Author: Yiqin Bao; E-mail: baoyiqin@njxzc.edu.cn 


\section{Bluetooth Technology}

Bluetooth technology[6-7] is a kind of radio technology which supports equipment with short-range (general $10 \mathrm{~m}$ ) communications. It can realize wireless information exchange between mobile phone and other equipment. Through the Bluetooth radio, it can be very convenient to get the device name, MAC address, and even the signal strength. Nowadays, the smartphones are all equipped with Bluetooth function, and have unified standard and interface.

\section{The System Overall Structure}

Automatic sign-in system based on Bluetooth technology, mainly consists of the server, the APP installed on teachers' mobile phone, the students' mobile phone with bluetooth opened, Bluetooth network and the Internet, as shown in Figure 1. At the first time, students should change the mobile phone name (with his own name), open the Bluetooth to release the radio signals; The server is responsible for inputting and management of student information; APP is responsible for carrying interface for the teachers' mobile phone connected to the server, and the receiving and processing of Bluetooth signal. When the teacher wants to call the roll, they need to download the student list from the server. Then choose class number to enter the main interface. Click start after, mobile phone to receive Bluetooth broadcast signal nearby. At the same time, students are asked to change the name of their mobile phone name and open Bluetooth. When the teacher gets one mobile phone Bluetooth broadcast, it automatically gets the name and MAC address. If there is a match between the device name and the one in the list. The corresponding field in the form will change. At the end, data can be submitted to the server when the 'submit' button is clicked.

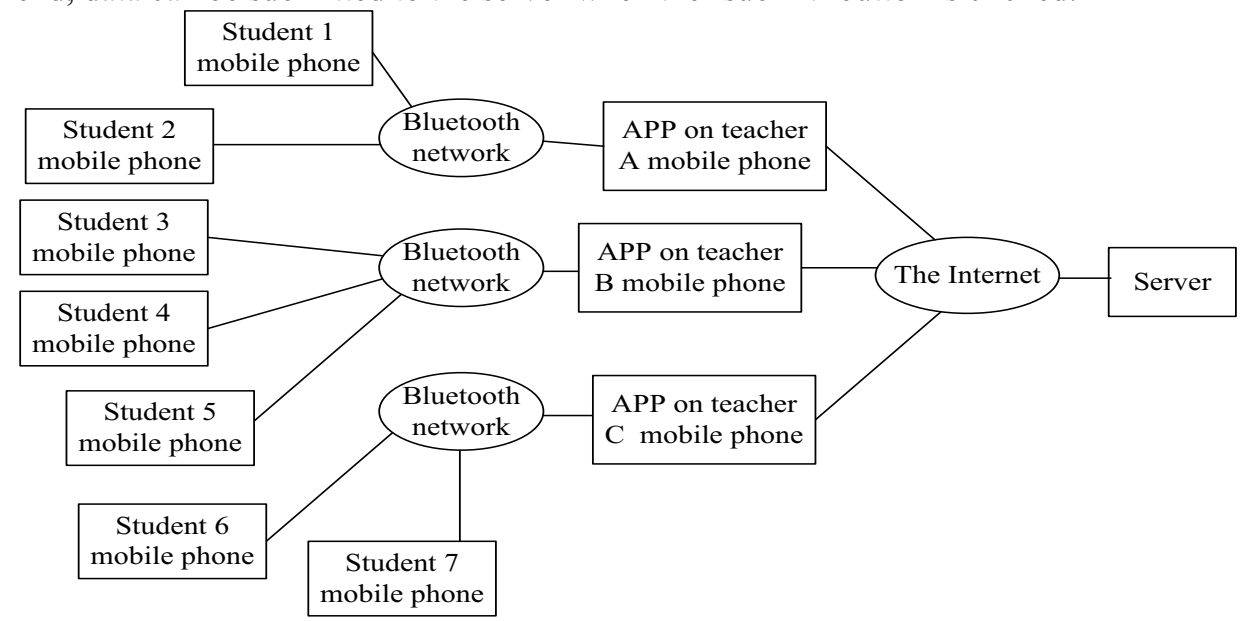

Figure 1. System architecture diagram 


\section{Design of the Server}

\subsection{Student Management Module}

Student management module is responsible for the management of student information, not only can choose class, query, add, modify and delete student information, but can also import student information directly from XLS documents.

\subsection{Interaction with the APP}

In the implementation of the interaction between server and mobile phone terminal, the main communicating method is adapting HTTP wireless communication, using a kind of lightweight data interchange format, JSON. JSON format has clear and concise hierarchical structure which makes it easy to read and write, as well as easy for machine to parse and generate. The transmission efficiency is high.

The Servlet in server receives the requests APP sends, make a series of responses, including the resolution of the JSON statement, extracting the corresponding parameters, the operation of the database, when necessary, to call JSONObject.put (Object key, Object value) method to create a JSON statement. In the system, the statement mainly includes some attributes of students returned to the mobile phone terminal.

\section{Design of the Server}

The homepage of the APP contains class choose button, 'download' button, 'delete' button and 'uri configuration' button, as shown in Figure 2. Before the teachers use the APP function to call the roll automatically, they need to set the IP address and port number. Then let the students open the phone Bluetooth and changed its names to their own names. At the same time click on the "start" button. Thus the sign-in process will be completed automatically.

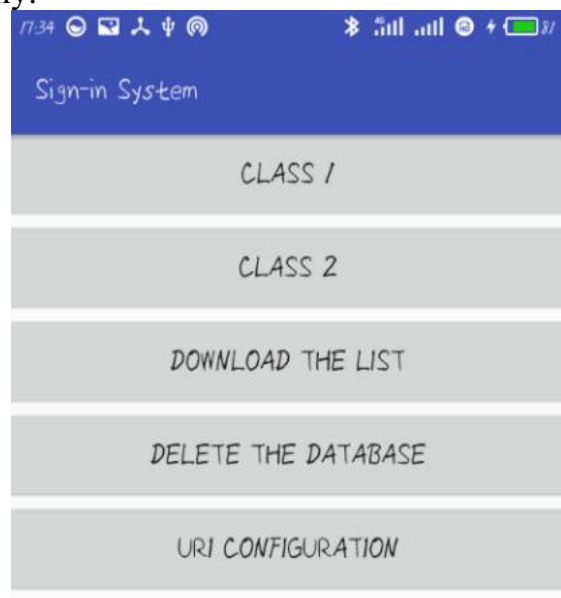

Figure 2. The homepage of the APP 


\subsection{Uri Configueration}

Because the data transmission using the HTTP protocol, teachers need to set the server URI (Uniform Resource Identifier) first, IP address and PORT number are needed. In the system, once submitted, the data will be stored in the SharedPreferences, thus there is no need to set the IP and PORT each time. The configuration interface is shown in Figure 3.

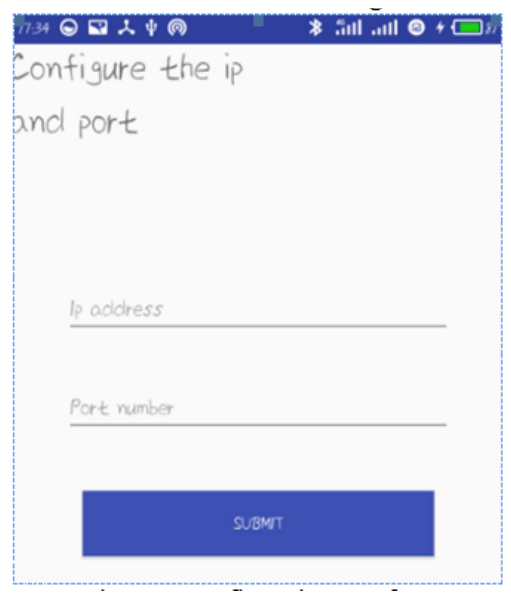

Figure 3. Configuration Interface

\subsection{Download the List}

When the curriculum is started or the list is changed, the teacher need download the list of the corresponding class first. In this process, the app gets connection to the server by the URI and transfer parameters. Then it will obtain the JSON statement returned, analyse it into student objects and store them in SQLite database.

\subsection{Choose Class}

Teacher taught more than one classes, while the list to download contains all the students list, so it needs to realize the class selection function in APP. When designing student information database, the class number is included as a property. Therefore, the students can be divided into the corresponding class, which is the base of the system.

\subsection{Call the Roll}

After entering the class interface, a table will be displayed, including student number, name, and state (initially empty) of all the students in the class. A BroadCastReceiver is set in the APP MainActivity to receive Bluetooth broadcast sent by the devices around. When receiving a Bluetooth broadcast signal, it will get the name and match it with the list of the class. If matched, a 'yes' will be written into the 'State' column. Thus, we complete 'calling the roll' automatically.

In the design of Bluetooth technology, the Bluetooth radio receiving equipment on the natural need to use Bluetooth devices, so we must first add Bluetooth permissions in the AndroidManifest.xml file, and then define the Bluetooth adapter access to access the native Bluetooth device. If bluetooth devices is discovered in after scanning, it will instantiate the discovered device, and use the object to get its name and other required attributes. 
Bluetooth permissions settings code as follows:

$<$ uses-permission android:name="android.permission.BLUETOOTH" $/>$

$<$ uses-permission android:name="android.permission.BLUETOOTH_ADMIN" $/>$

Bluetooth adapter instantiation code as follows:

private BluetoothAdapter mBluetoothAdapter;

mBluetoothAdapter $=$ BluetoothAdapter.getDefaultAdapter();

if(mBluetoothAdapter !=null) \{

if(!mBluetoothAdapter.isEnabled()) \{

Intent intent $=$ new Intent(BluetoothAdapter.ACTION_REQUEST_ENABLE);

startActivity(intent);

\}

mBluetoothAdapter.startDiscovery();

\}

else \{

textview.setText("Bluetooth device is no found! "); \}

if (action.equals(BluetoothDevice.ACTION_FOUND)) \{

BluetoothDevice

device=intent.getParcelableExtra(BluetoothDevice.EXTRA_DEVICE);

mTextView.append(device.getName ()$+$ " : " + device.getAddress ()$+" \backslash n ")$; \}

After clicking on the 'START' button, when discovering a matching device, corresponding fields in the table (and time) will be modified and saved in the SQLite, the name and address of all Bluetooth devices will also display under the table, as shown in Figure 4. Teachers can also clear the data for the next time to useit.

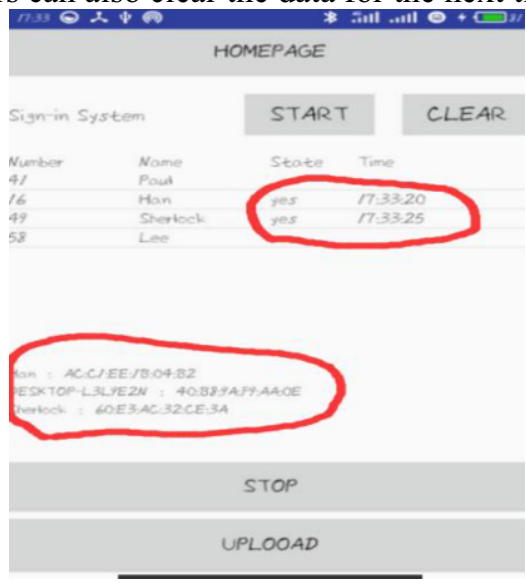

Figure 4. Automatic sign in interface

\subsection{Upload}

The way of uploading in this system is to get the students list from the SQLite to generate JSON format statement and transmit it to the server by HTTP protocol. The server will modify the database according to the JSON statement in, thus complete the uploading process. 


\section{The Comparison with Several Other Ways}

In order to illustrate the feasibility of the sign in system based on Bluetooth adopted in this paper, we compare RFID swipe card sign in, wireless routing sign in, face recognition sign in, fingerprint recognition sign in, etc. the comparison results are shown in Table 1. The results show that the check-in method used in this paper has better advantages.

Table 1. Comparison of different kinds of sign-in modes

\begin{tabular}{|l|l|l|}
\hline Solution & \multicolumn{1}{|c|}{ Advantages } & \multicolumn{1}{c|}{ Disadvantages } \\
\hline Credit card sign-in & $\begin{array}{l}\text { Faster recognition } \\
\text { speed and higher } \\
\text { stability }\end{array}$ & $\begin{array}{l}\text { The cost of the card is high and it } \\
\text { might get damaged and lost easily. } \\
\text { Data access can be affected by the } \\
\text { enviroment. }\end{array}$ \\
\hline Face recognition & $\begin{array}{l}\text { No need of users' } \\
\text { operation }\end{array}$ & $\begin{array}{l}\text { The recognition speed is slow and } \\
\text { the equipments are expensive. }\end{array}$ \\
\hline $\begin{array}{l}\text { Mobile phone } \\
\text { sign-in based on } \\
\text { wireless router }\end{array}$ & $\begin{array}{l}\text { The step is simple, the } \\
\text { operation is few and } \\
\text { the accuracy is high. }\end{array}$ & $\begin{array}{l}\text { Need to install WiFi to sign in, and } \\
\text { there is a limit to the number of } \\
\text { devices wireless router can } \\
\text { connect;Frequency bands of WiFi } \\
\text { generated by wireless router used to } \\
\text { sign in are close to each other, } \\
\text { which will interfere with each other, } \\
\text { affecting the public network }\end{array}$ \\
\hline $\begin{array}{l}\text { Fingerprint } \\
\text { recognition }\end{array}$ & $\begin{array}{l}\text { Medium speed of } \\
\text { recognition. }\end{array}$ & $\begin{array}{l}\text { The equipments need maintenance } \\
\text { and the cost is high. }\end{array}$ \\
\hline $\begin{array}{l}\text { Sign-in system } \\
\text { based } \\
\text { Bluetooth }\end{array}$ & $\begin{array}{l}\text { It's convenient and in } \\
\text { addition to mobile } \\
\text { phone } \\
\text { additional; } \\
\text { user-friendly }\end{array}$ & $\begin{array}{l}\text { Students need to modify the name of } \\
\text { mobile phone with that of } \\
\text { himself(herself). }\end{array}$ \\
\hline
\end{tabular}

\section{Conclusions}

This paper realizes the call sign based on Bluetooth technology and mobile Internet technology. It is a system of innovation with great advantages including clear train of thought, simple method and low cost. Due to its own factors, Bluetooth 4.0 has a range of just $10 \mathrm{~m}$. Sometimes the classroom is too large for the mobile phone to receive broadcast signals in time and the limitation will come into being. But with the development of Bluetooth 5.0 and the continuous progress of technology, the scope of application of Bluetooth technology will continue to expand, sign-in system based on Bluetooth will have great prospects for development.

\section{Acknowledgement}

This work is supported by Key topics of the '13th five-year plan' for Education Science in Jiangsu Province (B-b /2020/01/18). 


\section{References}

[1] Zhang Liang, Lingyun Feng, Wang Wen, Bi Tao. Design and implementation of experiment attendance system based on RFID [J]. Modern electronic technology, 2015 (1).

[2] Wang Weihong, Chen Xiaoliang. Design and implementation of mobile phone system based on wireless router[J]. Journal of Zhejiang University of Technology, 2016 (6).

[3] Jing Zhou, Quan Wei, Tang Jie, Yan Han. Sign-up system based on face recognition[J]. Software engineering, 2017 (5).

[4] ZHUO Ya-qian, OU Bo. Face Anti-spoofing Algorithm for Noisy Environment[J]. Computer Science, 2021, 48(6A): 443-447.

[5] WU Qing-hong, GAO Xiao-dong. Face Recognition in Non-ideal Environment Based on Sparse Representation and Support Vector Machine[J]. Computer Science, 2020, 47(6): 121-125.

[6] Chen Xi, Xu Peng, Xu Xiaolei. Development of embedded industrial control system based on Bluetooth communication technology [J]. Instrument technology and sensor, 2015 (1): 74-76.

[7] HONG Pengcheng, HUANG Yicai, YU Bin. Research on Bluetooth Encrypted Speech Synchronization for Mobile Communication Network. CEA, 2020, 56(13): 131-136. 